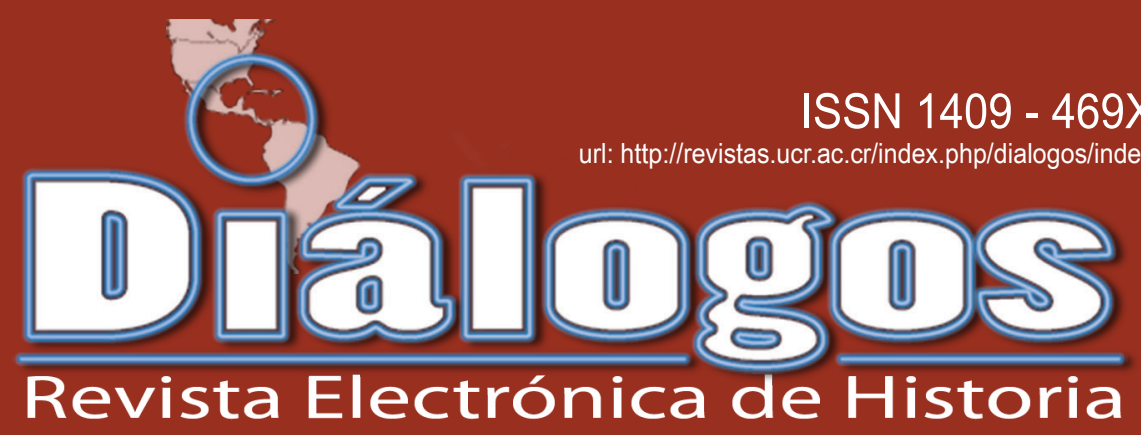

Escuela de Historia. Universidad de Costa Rica Volumen 14 especial en homenaje a Victor Hugo Acuña. Octubre 2013

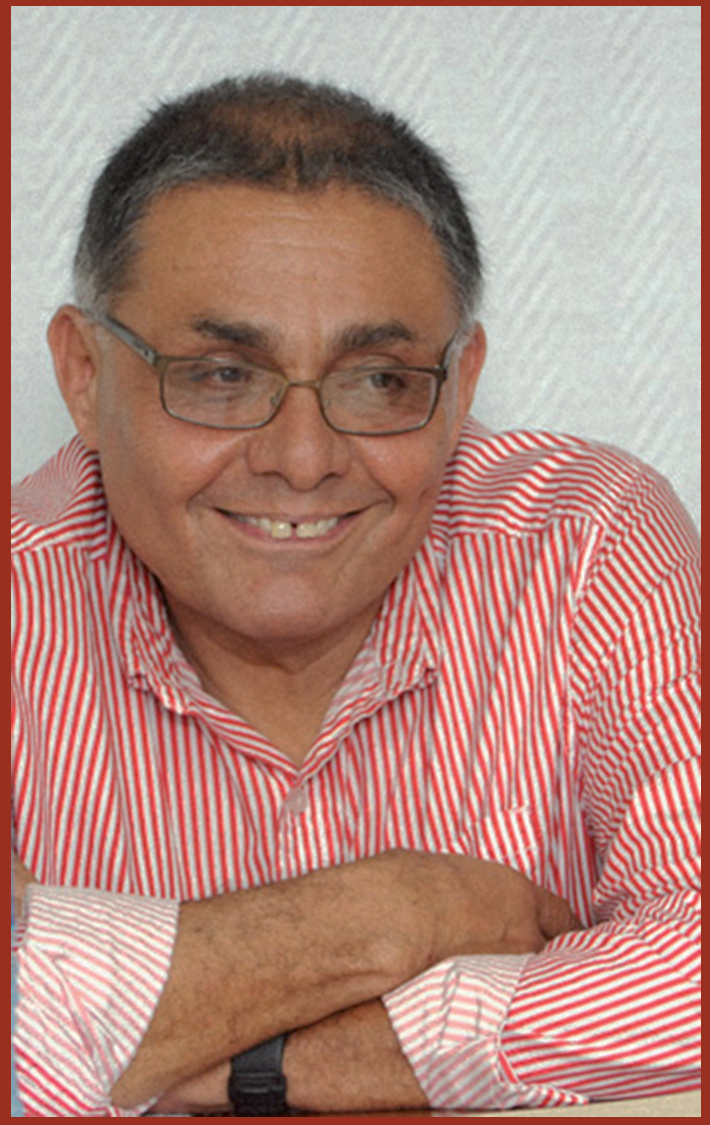

Director de la Revista: Dr. Juan José Marín Hernández juan.marinhernandez@ucr.ac.cr

Editor académico: Dr. Ronny Viales Hurtado - ronny.viales@ucr.ac.cr Editor técnico: M.Sc. Marcela Quirós G. - marcela.quiros@ucr.ac.cr 



\section{Miembros del Consejo Editorial:}

Dr. Juan José Marín Hernández, Catedrático. Director del Centro de Investigaciones Históricas de América Central. Universidad de Costa Rica. Costa Rica. juan. marin@ucr.ac.cr

Dr. Ronny Viales Hurtado. Catedrático. Historia Económica y Social. Universidad de Costa Rica. Director de la Escuela de Historia. Costa Rica. ronny. viales@ucr.ac.cr

Dr. David Díaz Arias: Catedrático. Historia Política, Director del posgrado de Historia y Docente de la Escuela de Historia, Universidad de Costa Rica, Costa Rica.david.diaz@ucr.ac.cr

MSc. Francisco Enríquez. Historia Social. Universidad de Costa Rica. Costa Rica. francisco.enriquez@ucr. ac.cr

Dra. Ana María Botey. Historia de los movimientos sociales. Universidad de Costa Rica. Costa Rica. abotey@gmail.com

\section{Miembros del Consejo Asesor Internacional:}

Dr. José Cal Montoya. Universidad de San Carlos de Guatemala.Guatemala. jecalm@correo.url.edu.gt

Dr. Juan Manuel Palacio. Universidad Nacional de San Martín. Argentina.jpalacio@unsam.edu.ar

Dr. Eduardo Rey. Universidad de Santiago de Compostela. España. ereyt@usc.es

Dr. Heriberto Cairo Carou. Departamento de Ciencia Política y de la Administración III Universidad Complutense de Madrid. España. hcairoca@cps.ucm.es

Dra. Rosa de la Fuente. Departamento de Ciencia Política y de la Administración III Universidad Complutense de Madrid. España. rdelafuente@cps. ucm.es

Dr. Javier Franzé. Departamento de Ciencia Política y de la Administración III Universidad Complutense de Madrid. España. javier.franze@cps.ucm.es

Dr. Jaime Preciado Coronado Departamento de Estudios Ibéricos y Latinoamericanos. Universidad de Guadalajara. México. japreco@hotmail.com
Dr. Gerónimo de Sierra. Vicerrector de la Universidade Federal da Integração Latino-Americana (UNILA) y Departamento de Sociología, Facultad de Ciencias Sociales de la Universidad de la República. Uruguay. geronimo@fcs.edu.uy

Dr. Antonio Palazuelos. Departamento de Ciencia Política y de la Administración III - Universidad Complutense de Madrid. España. palazuelosa@cps. ucm.es

Dr. Werner Mackenbach. Universidad Potsdam. Alemania. werner.mackenbach@uni-potsdam.de

Dr. Guillermo Castro. Ciudad del Saber Panamá. Panamá.gcastro@cdspanama.org

Dra. Natalia Milanesio. University of Houston. Estados Unidos.nmilane2@Central.UH.EDU

Dr. Ricardo González Leandri. Consejo Superior de Investigaciones Científicas - España. España. rgleandri@gmail.com

Dra. Mayra Espina. Centro de Estudios Psicológicos y Sociológicos, La Habana. Cuba.mjdcips@ceniai.inf.cu

Dra. Montserrat Llonch. Departamento de Economía e Historia Económica Universidad Autónoma de Barcelona. España.montserrat.llonch@uab.es

Dra. Estela Grassi. Universidad de Buenos Aires. Argentina. estelagrassi@gmail.com

Dra. Yolanda Blasco. Universidad de Barcelona. España. yolandablasco@ub.edu

Dr. Alfredo Falero. Departamento de Sociología. Universidad de la República. Uruguay. alfredof@adinet. com.uy

\section{Portada:}

Fotografía de Anel Kenjekeeva, Oficina de Divulgación Universidad de Costa Rica, publicada en http://www. ucr.ac.cr/noticias/2012/09/13/escuela-de-historia-rindehomenaje-a-victor-hugo-acuna.html

\section{Equipo Técnico Editorial:}

$\begin{array}{ll}\text { Editora Técnica: } & \text { M.Sc. Marcela Quirós Garita. } \\ & \text { marcela.quiros@ucr.ac.cr } \\ \text { Diagramación: } & \text { Cindy Chaves Uribe } \\ \text { Asistentes: } & \begin{array}{l}\text { Pablo Hurtado Granados } \\ \text { Maureen Méndez Montero }\end{array}\end{array}$


"Diálogos Revista Electrónica de Historia" se publica desde octubre de 1999.

\section{Diálogos está en los siguientes repositorios:}

Dialnet

http://dialnet.unirioja.es/servlet/

revista?tipo_busqueda=CODIGO\&clave_revista $=3325$

Latindex

http://www.latindex.unam.mx/larga.php?opcion=1\&folio=12995;

\section{UCRindex}

http://www.revistas.ucr.ac.cr/

\section{Scielo}

http://www.scielo.cl/

\section{eRevistas}

http://www.erevistas.csic.es/

\section{REDALYC}

http://redalyc.uaemex.mx/src/inicio/FrmBusRevs2.jsp?iEdoRev=2\&cvepai=11;

\section{LANIC}

http://lanic.utexas.edu/la/ca/cr/indexesp.html

Repositorio de Revistas Universidad de Costa Rica http://www.latindex.ucr.ac.cr/

Directorio y recolector de recursos digitales del

Ministerio de Cultura de España

http://roai.mcu.es/es/inicio/inicio.cmd

DOAJ Directory of open access \& Hybrid journals

http://www.doaj.org/doaj?func=byTitle\&hybrid=1\&query=D

Biblioteca de Georgetown

http://library.georgetown.edu/newjour/d/msg02735.html

Asociación para el Fomento de los Estudios Históricos

en Centroamérica

http://afehc. apinc.org/index.php?action=fi_aff\&id=1774

Universidad de Saskatchewan, Canadá

https://library.usask.ca/ejournals/view/1000000000397982

\section{Monografias}

http://www.monografias.com/Links/Historia/more12.shtml

\section{Hispanianova}

http://hispanianova.rediris.es/general/enlaces/hn0708.htm

Universidad del Norte, Colombia

http://www.uninorte.edu.co/publicaciones/memorias/enlaces.htm

Universidad Autónoma de Barcelona

http://seneca.uab.es/historia/hn0708.htm

Repositorio Invenia - Gestión del Conocimiento

http://www.invenia.es/oai:dialnet.unirioja.es:ART0000086144

\section{Enlace Académico}

http://www.enlaceacademico.org/biblioteca/

revistas-en-formato-digital-centroamerica/

\section{Electronic Resources}

http://sunzi1.lib.hku.hk/ER/detail/hkul/3987318

Revistas académicas en texto completo

http://web.prw.net/ vtorres/
Diálogos se anuncia en las siguientes

instituciones y sitios académicos:

Maestroteca

http://www.maestroteca.com/detail/553/dialogos-revista-electronica-de-historia.html

Biblioteca de Georgetown

http://library.georgetown.edu/newjour/d/msg02735.html

Asociación para el Fomento de los Estudios Históricos en Centroamérica

http://afehc. apinc.org/index.php?action=fi_aff\&id=1774

Universidad de Saskatchewan, Canadá

https://library.usask.ca/ejournals/view/1000000000397982

\section{Monografias}

http://www.monografias.com/Links/Historia/more12.shtml

\section{Hispanianova}

http://hispanianova.rediris.es/general/enlaces/hn0708.htm

Universidad del Norte, Colombia

http://www.uninorte.edu.co/publicaciones/memorias/enlaces.htm |

Universidad Autónoma de Barcelona

http://seneca.uab.es/historia/hn0708.htm

Repositorio Invenia - Gestión del Conocimiento

http://www.invenia.es/oai:dialnet.unirioja.es:ART0000086144

\section{Enlace Académico}

http://www.enlaceacademico.org/biblioteca

revistas-en-formato-digital-centroamerica/

\section{Electronic Resources}

http://sunzi1.lib.hku.hk/ER/detail/hkul/3987318

Revistas académicas en texto completo

http://web.prw.net/ vtorres/

La revista electrónica Diálogos es financiada por

Vicerrectoría de Investigación de la Universidad de Costa Rica
Dialnet - eRevistas

- UCRindex - Latindex -

REDALYC - DOJAC - Directorio

y recolector de recursos

digitales del Ministerio de

Cultura de España

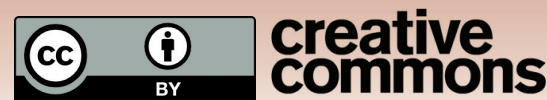





$$
\text { DOSSIER }
$$





\title{
DOSSIER HOMENAJE AL DR. VÍCTOR HUGO ACUÑA ORTEGA
}

David Díaz Arias

El 3 de septiembre del 2012, el Posgrado Centroamericano en Historia, el Centro de Investigaciones Históricas de América Central y la Escuela de Historia de la Universidad de Costa Rica le rindieron un homenaje al Dr. Víctor Hugo Acuña Ortega, destacado miembro del gremio de historiadores costarricenses. Un año después, el Dr. Acuña se convirtió en profesor emérito de la Escuela de Historia de la que se había jubilado unos años antes. El homenaje planificado fue una ocasión para subrayar el papel central que ha tenido el Dr. Acuña Ortega en la construcción de la historiografía centroamericana desde la mitad de la década de 1970 hasta el presente.

De esa influencia hay múltiples pistas y testimonios. Uno es el que se admira en una autobiografía que publicó hace varios años Iván Molina:

\begin{abstract}
Las figuras más importantes entre los docentes eran Carlos Rosés y Víctor Hugo Acuña... La docencia de Víctor Hugo Acuña, en óptimo contraste con la de Rosés, se distinguía por un derroche de pasión e imaginación, de desasosiego intelectual y de ironía en la crítica; entre mis compañeros se decía que era imposible asistir a una clase suya sin ver el pasado de distinta forma. La verdad de esa advertencia se vislumbraría en un futuro cercano: aunque publicó poco entre 1978 y 1981, él contribuyó decididamente a la investigación histórica a través de sus cursos de Colonial y Económica y social; en tales asignaturas, cultivó ideas cuya cosecha dejó a otros, en flagrante desacato del copyright (Molina, 1998).
\end{abstract}

En efecto, esa práctica que Molina ubicó a finales de los setentas e inicios de los ochentas, la ha repetido Acuña Ortega a lo largo de su destacada carrera como historiador. Por toda Centroamérica escucharán el eco de esa sentencia. Eso es justamente lo que queríamos celebrar en aquella ocasión con un homenaje a un verdadero maestro. Para hacerlo, organizamos un seminario en el que participaron el Dr. Ronny Viales Hurtado, el Dr. Juan José Marín Hernández, el M.Sc. George I. García, el Dr. Francisco Rojas y quien escribe esta presentación. La actividad, realizada en el miniauditorio de la Facultad de Ciencias Sociales de la Universidad de Costa Rica, estuvo todo el día repleta de estudiantes, historiadores y diversos científicos sociales de diversas edades. La idea que planteamos entonces fue releer los trabajos de Acuña Ortega, analizar su aporte a partir de ellos y proponer nuevas lecturas de las problemáticas construidas por él. 
El trabajo del Dr. Acuña Ortega todavía precisa de un ejercicio de análisis mayor. ${ }^{1}$ Por ahora, es posible advertir cuatro grandes patrones de análisis en su rica historiografía. En primer lugar, un inicial acercamiento a la historia económica que comenzó en la década de 1970 con estudios sobre el tabaco en el final de la época colonial en Costa Rica y sobre el comercio en el reino de Guatemala. A esos trabajos sucedió una etapa en la que Acuña Ortega se interesó por la historia de los obreros, los trabajadores urbanos y sobre los patrones del conflicto y la organización de los campesinos en Costa Rica en la primera mitad del siglo XX. Ese tipo de análisis también produjo aportes para la historiografía centroamericana y se valió de estrategias entonces novedosas como la historia oral. En un tercer momento, Acuña Ortega se interesó por la invención de las identidades nacionales en Centroamérica en el siglo XIX. Finalmente, su carrera se ha dirigido hacia el estudio de la memoria, especialmente en lo concerniente a la Guerra contra los filibusteros (1856-1857). En este dossier, incluimos dos de los estudios que se presentaron en aquel homenaje dedicado a Acuña Ortega y un trabajo bio-bibliográfico del homenajeado que se realizó en el 2008. Los otros trabajos, eventualmente, aparecerán también publicados. Asimismo, se incluye en este número la conferencia final de aquel seminario dedicado a su obra, que leyó el Dr. Acuña Ortega. Confiamos en que este dossier anime a más historiadores a revisitar la obra de Acuña Ortega y a encontrar en ella la fuente inspiradora que ha inspirado a muchos de sus discípulos.

\section{NOTAS}

1. Hay una recolección, ya desactualizada, de los títulos de su producción historiográfica en: Alvarado Barrientos y Mora Fernández, 2008.

\section{BIBLIOGRAFÍA}

Alvarado, V y Mora, A. (2008). Biobibliografía del Dr. Víctor Hugo Acuña Ortenga, historiador costarricense. Costa Rica: Escuela de Bibliotecología y Ciencias de la Información.

Molina, I. (1998). De un oficio antiguo y sin sentido. Ciencia social en Costa Rica. Experiencias de vida e investigación. Costa Rica: Editorial de la Universidad Nacional. 